\title{
Advantages and Disadvantages of Institutional Delivery and Home Delivery: A Qualitative Study in Northern Nigeria
}

\author{
Ryoko Sato ${ }^{1, *}$, Yoshito Takasaki ${ }^{2}$ \\ ${ }^{1}$ Harvard T. H. Chan School of Public Health, Harvard University, Boston, USA \\ ${ }^{2}$ Graduate School of Economics, University of Tokyo, Tokyo, Japan
}

Email address:

ryokos1226@gmail.com (R. Sato), rsato@hsph.harvard.edu (R. Sato), takasaki@e.u-tokyo.ac.jp (Y. Takasaki)

${ }^{*}$ Corresponding author

\section{To cite this article:}

Ryoko Sato, Yoshito Takasaki. Advantages and Disadvantages of Institutional Delivery and Home Delivery: A Qualitative Study in Northern Nigeria. European Journal of Preventive Medicine. Vol. 9, No. 1, 2021, pp. 19-24. doi: 10.11648/j.ejpm.20210901.14

Received: January 22, 2021; Accepted: February 17, 2021; Published: February 27, 2021

\begin{abstract}
Background: Nigeria faces high maternal and infant mortality; and Nigeria is one of the two countries, with India, that account for one-third of global child mortality. Many deaths could be prevented by institutional delivery, yet many women especially in developing countries still deliver a baby at home. Nigeria is not an exception; it faces low rate of institutional delivery, only $35.8 \%$. Objective: To identify why many women do not deliver at a clinic, we compare the advantages and disadvantages of each place - home and clinic - for childbirth. Method: We conducted a qualitative study among 86 women in northern Nigeria. Data were analyzed using inductive content analysis. Result: Among women who delivered a baby at home, the main barriers to institutional delivery include misconception about the importance, unpleasant clinic environment, and perceived harsh attitudes of health staff. One advantage of home delivery is the emotional support from family. Most women who delivered at clinic are satisfied with their experiences with no complaint about harsh staff attitude. The main disadvantages of clinic delivery is the condition of the clinic. Conclusion: The low quality of care at the health clinics is a concern and should be improved. Providing women with emotional support and pleasant environment at clinic are two feasible and potentially effective options.
\end{abstract}

Keywords: Barriers to Institutional Delivery, Advantages of Home Delivery, Northern Nigeria, Emotional Support

\section{Introduction}

Despite recent advances in the reduction of maternal and infant mortality across the globe, the levels in sub-Saharan Africa remain unacceptably high. Sub-Saharan Africa accounts for 66 percent of global maternal deaths, with Nigeria, our study site, alone accounting for 19 percent [1]. In addition, about 80 percent of child mortality occurs in Sub-Saharan Africa and South Asia, and Nigeria is one of the two countries, with India, that account for one-third of global child mortality [2].

Most of these deaths could be prevented with institutional delivery [1-3]. However, despite the importance of institutional delivery, many women in developing countries still deliver a baby at home. For example, institutional delivery accounts for only $35.8 \%$ of births in Nigeria [4].

Extant qualitative studies have intensively examined barriers to institutional delivery in developing countries [5-7]. The main approach in these studies is to ask respondents about the perceived barriers to institutional delivery. They found common barriers such as lack of access to clinics, socio-cultural factors, risk perceptions about childbirth, and supply-side issues.

However, to identify why many women do not deliver at a clinic, we need to compare the advantages and disadvantages of each place - home and clinic - for childbirth, instead of only assessing the disadvantages of home delivery, which is a common approach in the literature. Then, policies can be designed to increase the comparative advantages of clinic delivery by strengthening factors that are advantages of clinic delivery and mitigating factors that are disadvantages of clinic delivery, while incorporating advantageous factors of 
home delivery to clinic environments.

We conducted a qualitative interview among women in northeastern Nigeria in May 2015 to learn about their experiences of, beliefs about, and attitudes toward childbirth. In particular, we not only assessed the perceived barriers to clinic delivery among women who delivered at home, but also did we assess perceived advantages of home delivery. We also approached women who had delivered at clinics and asked about their negative experiences at those clinics during their childbirths as well as perceived advantages of clinic delivery.

We found that many women who had delivered at home had unfavorable experiences of home delivery because their homes did not provide a pleasant environment. Furthermore, the majority of them perceived that home delivery is less safe than clinic delivery. Nevertheless, the timing of birth, lack of adequate information on the importance of clinic delivery, and concerns about clinic environment, including health staffs' attitudes, prevent them from delivering at a clinic. On the other hand, we found that the emotional support that women receive at home is one of the major advantages of home delivery. As with the women who had delivered at home, among the women who had delivered at a clinic, one of the main complaints was also about a less-thancomfortable/pleasant environment.

To promote the take-up of clinic delivery, there is a need to improve emotional support given at clinics and clinic environment in terms of hygiene and comfort.

\section{Methods}

\subsection{Sample}

To obtain an in-depth understanding of experiences of, attitudes toward, and beliefs about pregnancy and childbirth among women who had experienced childbirth, we conducted a qualitative research comparing women who had delivered a baby at home and women who had delivered a baby at a clinic. We asked their experiences about their first childbirths, using the questionnaire the author prepared.

The study, conducted in May 2015, was carried out in Jada Local Government Area (LGA) of Adamawa state, in northeastern Nigeria, with ethical approval from Health Research Ethical Committee at Adamawa State Ministry of Health.

Our qualitative study employed two approaches. The first approach was individual qualitative interviews, conducted in 6 villages, randomly selected from villages within the catchment area of the Jada Primary Health Care Clinic (PHCC). The second approach was focus group interviews among women who visited the Jada PHCC. We conducted 6 focus group discussions in total (30 women in total).

Data were created based on the field notes that were prepared by interviewers during the interview, and were analyzed using inductive content analysis. We combine the individual interview sample (56 women) and the focus group sample (30 women), which makes the total sample of 86 women.

\subsection{Demographic Characteristics}

Table 1 presents the descriptive statistics of women. 52 women had delivered a first child at home, while the remaining 34 had delivered a first child at a clinic. Respondents were on average 28 years old. Over half of the respondents were Muslim.

Table 1. Demographic characteristics of women by the place of childbirth $(n=86)$.

\begin{tabular}{llll}
\hline & \multicolumn{3}{l}{ Average (\%) } \\
\cline { 2 - 4 } & home & clinic & Difference (p-value) \\
\hline Age & 29.58 & 27.64 & 0.182 \\
Religion & & & \\
Christian & 0.39 & 0.56 & 0.216 \\
Muslim & 0.61 & 0.44 & 0.216 \\
\# of childbirths & 18.97 & 20.08 & 0.154 \\
\# of children who died & 4.39 & 3.24 & 0.073 \\
Education & 0.81 & 0.60 & 0.473 \\
none & & & \\
primary & 0.47 & 0.22 & 0.094 \\
secondary and above & 0.17 & 0.72 & 0.000 \\
$\begin{array}{l}\text { Occupation } \\
\text { housewife }\end{array}$ & 0.37 & 0.06 & 0.015 \\
farmer & 0.76 & 0.50 & 0.052 \\
nonfarm job & 0.17 & 0.13 & 0.639 \\
$\begin{array}{l}\text { Husband's occupation } \\
\text { farmer }\end{array}$ & 0.07 & 0.38 & 0.001 \\
businessman & 0.33 & 0.36 & 0.162 \\
wage earner & 0.33 & 0.32 & 0.911 \\
\hline
\end{tabular}

Women experienced their first childbirth at about 19. On average, women had experienced 3 to 4 childbirths, and 0.7 children had died during delivery or immediately after birth. The number of child births was significantly higher among mothers who delivered at home (4.39) as compared to mothers who delivered at a clinic (3.24). The number of children who died was insignificantly higher among mothers who delivered at home $(0.81)$ as compared to mothers who delivered at a clinic (0.60).

Among women who delivered at home, almost half (47 percent) did not have any education, while only 17 percent of them had higher education than primary level. On the other hand, the majority (72 percent) of respondents who delivered at a clinic had completed higher education, while only 22 percent of them had no education.

Most of respondents were housewives, but the proportion of housewives was significantly higher among those who delivered at home (76 percent) than among those who delivered at a clinic (50 percent), while the proportion of women with nonfarm job was lower among those who delivered at home ( 7 percent) than among those who delivered at a clinic (38 percent). Husbands' occupations were almost equally divided between farming, business, and wage earners.

Although we cannot speak for the representativeness of our sample in the study area, the data suggests that women who deliver at home deliver more children, are less educated, 
and are more likely to be housewives than women who deliver at a clinic.

\section{Results}

\subsection{Concerns About, Preparation for, and Problems with Childbirth}

This section summarizes the concerns and problems around childbirths. Regardless of the place of delivery, women have great concerns about childbirth.

Concerns about childbirth

Many women have fears about childbirth. Concerns are mainly focused on the serious health consequences for the women and the newborn babies:

"Maybe something might happen to me during the labor and I do not know if I can survive. Maybe I will deliver something that is not a human-being". (Zainab, Tudun Wada).

Other concerns specifically among women who delivered at a clinic include concerns about health staffs' attitudes:

"I do not know if I would be in good hands with health staffs during labor." (Martha, Jada town)

Preparation for childbirth

Both women who delivered a baby at home and those who delivered at a clinic prepared for childbirth in similar ways, by buying items necessary for the delivery, such as soap, razor blade, and sanitary pads. Women who delivered at a clinic prepared similar items because health staffs commonly encourage women to bring items for the delivery themselves.

Problems with childbirth

All the women, regardless of place of delivery, have similar types of problems during and after a birth. These problems include moderate physical discomfort such as dizziness and abdominal pain, while retained placenta and loss of blood during labor were also raised by some women. These problems are often considered critical if women deliver at home without skilled assistance.

\subsection{Childbirth from the Perspective of Women Who Chose Home Delivery}

This section summarizes the beliefs and attitudes that prevent women from utilizing a clinic for childbirth. We asked women who had delivered a baby at home 1) the reasons of not delivering at a clinic, 2) how much paid for delivery assistance, 3) what not liked about home delivery, 4) what liked about home delivery, and 5) place they considered safer for the birth - home or clinic.

Why did you not deliver at clinic?

Timing: Childbirth is a time-sensitive event. Many women identified the timing issue as the major barrier to the utilization of a clinic for childbirth. One woman, for example, said

"Before I realized, the baby started to come down and there was no time to go to the clinic then". (Hauw'au, Jada town)

Some also said that lack of transportation at that time in the middle of the night prevents the institutional delivery.
Misconception about the importance of institutional delivery: Some women said that they did not deliver at a clinic because they did not have any problem during their pregnancy. This statement indicates that the awareness of the importance of institutional delivery or the risk around childbirth among women is not sufficient. Some also said that they did not deliver at a clinic because they have health staff near their house. While it is important that women are assisted by professional personnel at the time of childbirth, institutional delivery is critical for safe delivery because clinics are well equipped with devices and medicines. The results show that these women are not well aware of the importance of the place of childbirth.

Clinic condition/health staffs' attitude: While some listed the distance to the clinic, monetary expense, and the position in which women deliver a baby at a clinic as barriers to institutional delivery, many complained that health staff are harsh when women deliver at a clinic. However, their beliefs are often not based on their own experience but rather, on stories they have heard from their friends about how harsh and rude health staff are.

How much did you pay for the assistant for delivery (home)?

Delivery at home is not free. The majority of the women (67 percent) who had delivered a baby at home said that they paid those who assisted at the births at home. Out of women who paid, half of them paid in-kind compensations such as wrappers and soap, while the other half paid money, on average 1,280 Naira (about 4 USD; 1USD = N320 in May 2015).

What did you not like about the place of delivery (home)?

The most common dislike about home delivery was the bad smell of the place at the time of delivery. Through this study, we found that many women deliver in the bathroom. Then they complained about the smell of the bathroom and of blood at the time of labor.

The second most common answer was that everything about childbirth at home is good. The level of awareness of the importance of institutional delivery is low.

The third most common answer was that nothing about childbirth at home is good, due to reasons such as that the place is not clean, hot, and without privacy. This statement indicates that they might have liked to deliver at a clinic but due to some constraints they could not.

What did you like about the place of delivery (home)?

Emotional support: Many women said that childbirth at home was good because they could get emotional support from their relatives and assistants. One woman said:

"I like it because I can get care and gentle words, while at the clinic, health workers are harsh and not friendly". (Focus group, Jada town)

Privacy: However, others do not like being in an environment where any family members or neighbors can enter the room and thus prefer to deliver at a clinic.

Which place is safer for a delivery - clinic or home?

Even among women who delivered at home, the majority think that delivering at a clinic is safer than at home. Sixty percent of the respondents said that delivery at a clinic is 
safer because the clinics are more prepared to deal with an emergency situation; they have medicines, equipment, and professional health staffs. One woman said:

"Giving birth in the clinic can save the lives of the baby and the mother because health staffs monitor us and give us an injection if there is a problem". (Amina, Bobda)

The remaining 40 percent of women think that delivering at home is safer. The dominant reason for this belief is that they can obtain the care that they want at home. This is consistent with the observation that one of the main factors they liked about the home delivery was emotional support. Amina from Bobda said:

"Home delivery is safer. At the clinic, staffs do not attend to me on time and make me go through the pain alone. But at home, I have old women who care for me".

Another woman said:

"At home the assistant holds my back during the childbirth but at the clinic health staffs do not care and they abuse women .... The nurses abuse women by saying that they are enjoying themselves with their husbands, that is why they get pregnant". (Fatimatu, Bobda)

\subsection{Childbirth from the Perspective of Women Who Chose Clinic Delivery}

This section summarizes the beliefs and attitudes that motivate women to utilize a clinic at the time of childbirth. We also asked about their negative experiences at a clinic at the time of childbirth. We asked women who had delivered a baby at a clinic 1) the reasons why they had delivered a baby at clinic, 2) how much paid to the health clinic, 3) what disliked about clinic delivery, 4) what liked about clinic delivery, and 5) place they felt is safer for delivery - home or clinic.

Why did you decide to deliver at a clinic?

Risk: Many women recognized the importance of institutional delivery because it is safer and they can get proper care in case there is any problem during labor. One woman said:

"I delivered at the clinic because I can get more proper care from nurses and doctors". (Blessing, Jada town)

Own condition/experiences: Women might tend to deliver at clinic if they had observed any problems during their past delivery, or during the current pregnancy.

"I always had problems like retained placenta in past deliveries, and so now I always deliver with health staffs". (Benice, Tudun Wada)

Peers' influence: Their peers can influence their decisions. If peers' experience at a clinic is positive, women might follow their behaviors. Likewise, if women observe problems of delivery at home, they might deviate from home delivery.

"When my friend delivered at the clinic, I liked the way health staffs handled her, so I decided to deliver there too, after I discussed it with my husband". (Bilkisu, Nassarawo)

How much did you pay for the assistant for delivery (clinic)?

Although we observed that many women who deliver at home also pay for the service they receive, the amount that women pay for an institutional delivery is higher than that for a home delivery. The payment for an institutional delivery is entirely monetary. The average amount they pay for the institutional delivery is N1,690 (about 5.4 USD). Although officially, any institutional delivery at public clinics that does not involve complication should be free of charge, health clinics charge a significant amount of money for the service they provide to women.

What did you not like about the place of delivery (clinic)?

About half of the women said that everything was fine at the clinic. Others had various complaints such as that the bed at the clinic was not comfortable, that they suffered from mosquito bites at the clinic, and that the clinic was not clean. Some also identified the transportation to the clinic and the monetary costs for the service as the factors they do not like about using a clinic for childbirth.

What did you like about the place of delivery (clinic)?

Despite the common belief held among women who deliver at home that health staff are very harsh, many women who delivered at a clinic had positive experiences interacting with health staff. They appreciated the caring attitude of health staff during the labor.

"The way the staff attended to me; I liked it and felt comfortable". (Blessing, Jada town)

"The staff were hard working and kind". (Aishatu, Jada town)

Many women also acknowledged that they liked the clinic because they could receive the proper care from the professional staff.

Which place is safer for delivery - clinic or home?

All of the women who delivered at a clinic said that delivering at a clinic is safer than at home.

\section{Discussion}

This study aimed at understanding women's experiences with, attitudes toward, and beliefs about childbirth. We identified perceived advantages and disadvantages of the place of delivery they chose (clinic or home).

\subsection{Concerns About, Preparation for, and Problems with Childbirth}

Women in general have similar concerns about their and their babies' safety during the labor. They prepared well by purchasing delivery items ahead of time. Yet, many women encountered problems; some of which can critically endanger the mother's health.

\subsection{Experiences from Home Delivery}

Among women who delivered a baby at home, the timing of labor, misconception about the importance of institutional delivery, the unpleasant environment of the clinic, and beliefs about the harsh attitudes of health staff are the main reasons why they did not deliver at a clinic. Even though they delivered at home, they still paid some compensation to the assistant who helped them for the delivery. 
The condition under which women deliver at home is often not pleasant, with unpleasant odor and unhygienic condition. Many do not prefer delivering a baby at home. On the other hand, however, still many other women say that everything is good about home delivery.

Women who deliver at home state that the emotional support they receive at the time of childbirth is one of the reasons why they like home delivery.

Even though they deliver at home, more than half of them acknowledge that delivering at a clinic is safer. This perception indicates that they are constrained to have a home delivery against their wishes. However, it should also be noted that many other women think that home delivery is safer.

\subsection{Experiences from Clinic Delivery}

Women who delivered a baby at a clinic have high perceived risks of childbirth. The amount of payment for the service they receive is higher for the clinic delivery than that for home delivery.

About half of the women who delivered at a clinic are satisfied with the clinic delivery. Surprisingly, no one complained about the harsh attitude of health staff. Rather, many women stated that they had positive experiences of interacting with health staff. The common belief that health staff are harsh might be derived from occasional rare events. All the women who delivered at a clinic think that the clinic delivery is safer than home delivery.

\section{Conclusions}

This study aims to deepen our understanding of the underlying factors for the low take-up of institutional delivery in northeastern Nigeria. The evidence is limited in the area but it is important to evaluate perceptions among the population in the region.

This study revealed perceptions among women in this region, using the unique data on perceived barriers around childbirth. We investigated experiences with, attitudes toward, and beliefs about childbirth among women. In particular, we identified perceived advantages and disadvantages of home delivery and clinic delivery through the qualitative study.

We find that, regardless of the place of delivery, women encounter similar problems such as retained placenta and bleeding during and right after labor. Although we might expect that women who deliver at a clinic face fewer problems, that are not observed in our study. This result might indicate the low quality of care at the health clinics, which should be improved. Our findings are mostly consistent with findings from previous studies [8-15].

We find that many women still deliver at home even though they find it less safe and more unpleasant than clinic delivery, due to constraints such as the timing, the lack of adequate information on the importance of clinic delivery, and the concerns about the clinic environment including health staffs' attitude. One of the main perceived advantages of home delivery is emotional support, which clinic staff should be encouraged to provide to increase the take-up of institutional delivery.

Among women who deliver at a clinic, one of the main disadvantages of clinic delivery is the condition of the clinic. To promote the utilization of clinic at the time of childbirth in a sustainable way, we should ensure that the clinic offers a pleasant environment in terms of hygiene and privacy for women.

\section{References}

[1] WHO, UNICEF, UNFPA, World Bank Group, et al. Trends in Maternal Mortality: 1990 to 2015. Geneva, World Health Organization, 2015.

[2] United Nations Inter-agency Group for Child Mortality Estimation (UN IGME). Levels \& Trends in Child Mortality: Report 2017, Estimates Developed by the UN Inter-agency Group for Child Mortality Estimation. United Nations Children's Fund, New York, 2017.

[3] Kerber K, E de Graft-Johnson J, Bhutta Z, Okong P, et al. Continuum of care for maternal, newborn and child health: from slogan to service deliver. Lancet. 2007; 370: 1358-69. doi: 10.1016/S0140-6736(07)61578-5.

[4] National Population Commission (NPC) [Nigeria] and ICF International. Nigeria Demographic and Health Survey 2013. Abuja, Nigeria, and Rockville, Maryland, USA: NPC and ICF International, 2014.

[5] Vellakkal S, Reddy H, Gupta A, Chandran A, et al. A qualitative study of factors impacting accessing of institutional delivery care in the context of India's cash incentive program. Social Science and Medicine. 2017; 178: 55-65.

[6] Wilunda C, Quaglio G, Putoto G, Lochoro P, et al. A qualitative study on barriers to utilisation of institutional delivery services in Moroto and Napak districts, Uganda: implications for programming. 2014; 4 (14) 259 doi: 10.1186/1471-2393-14-259.

[7] Wilunda C, Scanagatta C, Putoto G, Takahashi R, et al. Barriers to Institutional Childbirth in Rumbek North County, South Sudan: A Qualitative Study. PLOS ONE. 2016; 11 (12): e0168083. https://doi.org/10.1371/journal.pone.0168083

[8] Dahab, Rana, and Dikaios Sakellariou. "Barriers to Accessing Maternal Care in Low Income Countries in Africa: A Systematic Review." International journal of environmental research and public health 17, no. 12 (2020): 4292.

[9] Tey, Nai-Peng, and Siow-li Lai. "Correlates of and barriers to the utilization of health services for delivery in South Asia and Sub-Saharan Africa." The Scientific World Journal 2013 (2013).

[10] Kyei-Nimakoh, Minerva, Mary Carolan-Olah, and Terence V. McCann. "Access barriers to obstetric care at health facilities in sub-Saharan Africa-a systematic review." Systematic reviews 6, no. 1 (2017): 1-16.

[11] Hernandez, Bernardo, Danny V. Colombara, Marielle C. Gagnier, Sima S. Desai, Annie Hakenstad, Casey Johanns, Claire R. McNellan et al. "Barriers and facilitators for institutional delivery among poor Mesoamerican women: a cross-sectional study." Health policy and planning 32, no. 6 (2017): 769-780. 
[12] Harahap, Nabila Clydea, Putu Wuri Handayani, and Achmad Nizar Hidayanto. "Barriers and technologies of maternal and neonatal referral system in developing countries: a narrative review." Informatics in Medicine Unlocked 15 (2019): 100184.

[13] Limwattananon, Supon, Viroj Tangcharoensathien, and Supakit Sirilak. "Trends and inequities in where women delivered their babies in 25 low-income countries: evidence from Demographic and Health Surveys." Reproductive Health Matters 19, no. 37 (2011): 75-85.

[14] Hasan, Md Mehedi, Ricardo J. Soares Magalhaes, Yaqoot
Fatima, Saifuddin Ahmed, and Abdullah A. Mamun. "Levels, Trends, and Inequalities in Using Institutional Delivery Services in Low-and Middle-Income Countries: A Stratified Analysis by Facility Type." Global Health: Science and Practice (2021).

[15] Bohren, Meghan A., Erin C. Hunter, Heather M. Munthe-Kaas, João Paulo Souza, Joshua P. Vogel, and A. Metin Gülmezoglu. "Facilitators and barriers to facility-based delivery in low-and middle-income countries: a qualitative evidence synthesis." Reproductive health 11, no. 1 (2014): 1-17. 